\title{
Analysis of flow characteristics in the tail reach of Ganjiang River, Poyang Lake in China
}

\author{
Hua $\mathrm{Ge}^{1, *}$, Chunyan Deng ${ }^{1}$ \\ ${ }^{1}$ Changjiang River Scientific Research Institute, Wuhan, Hubei, 430010, China
}

\begin{abstract}
At present, the traffic pressure of Nanchang city, the capital of Jiangxi province, is increasing. The increasing demand for the construction of river crossing channel requires strengthening the research on the flow characteristics of the tail reach of Ganjiang River where Nanchang is located. In this paper, the south section of Nanchang city at the tail end of Ganjiang River was taken as the research object. By applying the plane two-dimensional flow mathematical model as the technical means, and using the latest measured topographic and hydrological data in 2020 to verify the model, the flow movement characteristics of the studied river section were calculated and analysed. The results show that the water surface gradient is slow, the flow field is smooth, the flow velocity is small, and the sediment is easy to deposit in this reach, which is easy to threaten the flood control of the river section.
\end{abstract}

\section{Introduction}

Nanchang, the capital city of Jiangxi Province, is located at the end of Ganjiang River. In recent years, Nanchang has entered a period of rapid motorized growth, and the number of motor vehicles in 2019 had increased by about $11.2 \%$ over the previous year. At present, under the guidance of the urban development pattern of "one river on both sides of the river" in Nanchang City, the layout of river crossing channels in the north of the city is relatively perfect. From north to south, a river crossing channel system of "5 bridges +1 tunnel" composed of Hero Bridge, Bayi Bridge, Honggu tunnel, Nanchang Bridge, Chaoyang Bridge and Shengmi Bridge has been built. The density and traffic capacity of river crossing channels can basically meet the requirements of each area in the north of the city River traffic demand. However, there is only one river crossing channel of Shengmi Bridge in the south of the city, and the traffic supply across the river in the south of the city is far lower than the traffic demand across the river (see Figure 1). With the rapid development of Jiulong lake, Liantang and other groups in the future [1], the demand for river crossing travel in the south of the city will also increase rapidly. Shengmi Bridge has already been unable to meet the needs of residents to cross the river. The contradiction between the supply and demand of the traffic across the river in the south of Nanchang city will be further intensified. It is urgent to strengthen the layout and construction of the South River Crossing channel to relieve the traffic pressure in the south.

Bridge construction has different degrees of influence on flood and river regime, and the flow characteristics of river channel in turn have an impact on bridge construction [2-4]. In order to ensure the rationality, safety and economy of the bridge construction, we must consider the flow pattern and scouring and silting characteristics of the river to be crossed, and adjust measures to local conditions [2-3]. Based on a flatten two-dimensional flow mathematical model, the flow characteristics of Nanchang reach at the tail end of Ganjiang River was calculated and analysed in this paper, which will provide technical support for the construction of river crossing bridges in the south of Nanchang city.

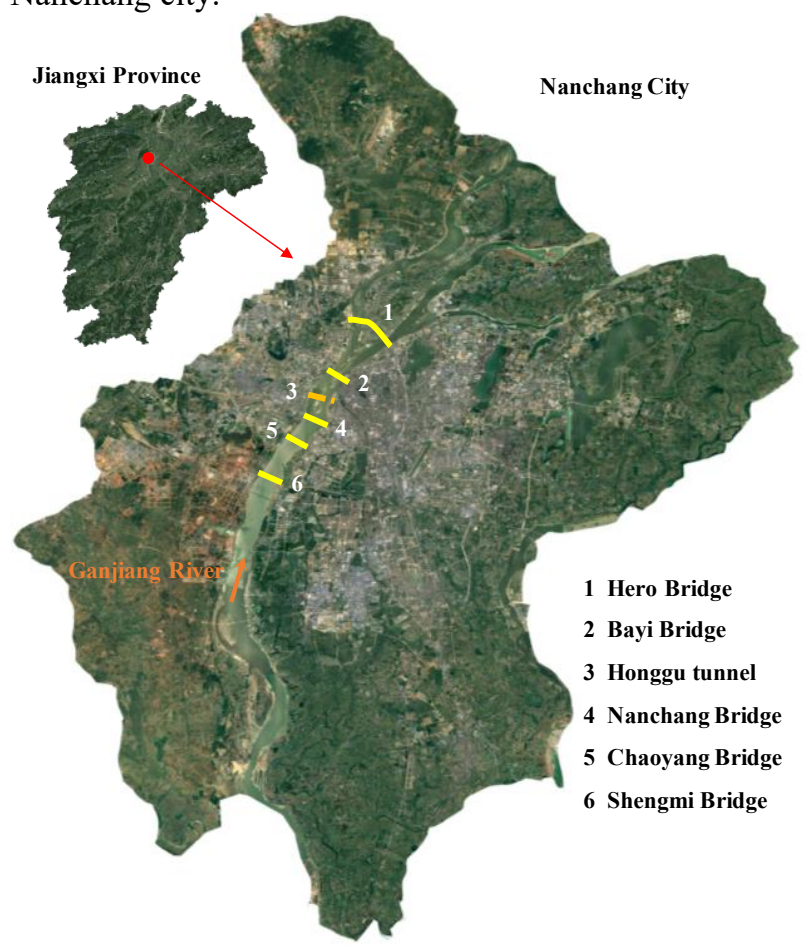

Fig. 1. Distribution of crossing channels in Nanchang City.

\footnotetext{
* Corresponding author: gh-102@126.com
} 


\section{Study area, method and data}

\subsection{Study area}

Considering the actual demand of bridge construction in the south area of Nanchang City, the study area is determined as the upper section of Nanchang section at the tail end of Ganjiang River, starting from Dongwu village and down to Bayi Bridge. The shape of the studied river section is straight and slightly curved, with very small curvature. The width of the channel is generally between $1 \mathrm{~km}$ and $2.5 \mathrm{~km}$, shown as Figure 2 .

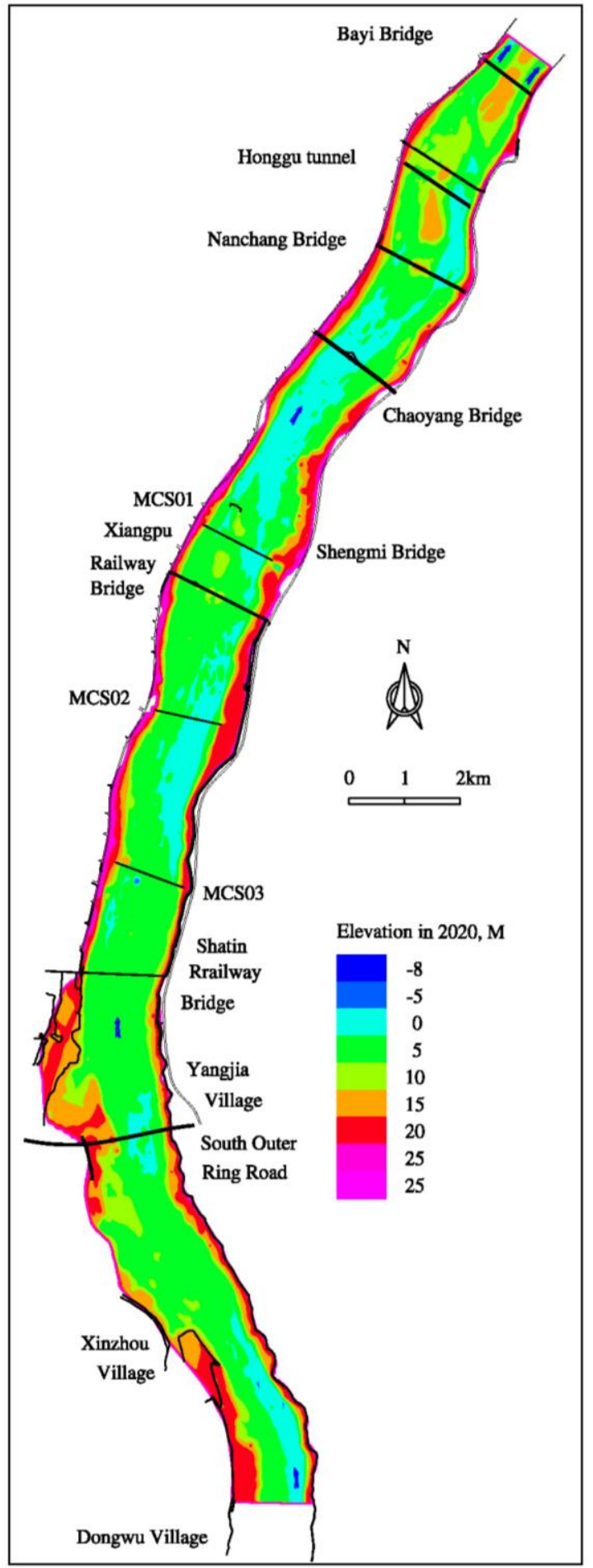

Fig. 2. Map of the study river reach.

\subsection{Method}

A flatten two-dimensional flow mathematical model is used in this paper. The governing equations in rectangular coordinates are as follows:

$$
\begin{gathered}
\frac{\partial Z}{\partial t}+\frac{\partial u H}{\partial x}+\frac{\partial v H}{\partial y}=0 \\
\frac{\partial u H}{\partial t}+\frac{\partial u u H}{\partial x}+\frac{\partial v u H}{\partial y}=-g \frac{n^{2} \sqrt{\left(u^{2}+v^{2}\right)}}{H^{\frac{1}{3}}} u-g H \frac{\partial Z}{\partial x}+v_{T} H\left(\frac{\partial^{2} u}{\partial x^{2}}+\frac{\partial^{2} u}{\partial y^{2}}\right) \\
\frac{\partial v H}{\partial t}+\frac{\partial u v H}{\partial x}+\frac{\partial v v H}{\partial y}=-g \frac{n^{2} \sqrt{\left(u^{2}+v^{2}\right)}}{H^{\frac{1}{3}}} v-g H \frac{\partial Z}{\partial y}+v_{T} H\left(\frac{\partial^{2} v}{\partial x^{2}}+\frac{\partial^{2} v}{\partial y^{2}}\right)
\end{gathered}
$$

Where: $H$ is the water depth, $u$ and $v$ are the velocity in the $x$ and $y$ directions, $Z$ is the water level; $n$ is the Manning roughness coefficient; $V_{T}$ is the turbulent viscosity coefficient, $g$ is the gravity acceleration.

According to the transformation relationship between orthogonal coordinate system and rectangular coordinate system, the above equations (1) to (3) can be transformed into those in orthogonal coordinate system:

$$
\begin{gathered}
\frac{\partial Z}{\partial t}+\frac{1}{C_{\xi} C_{\eta}}\left[\frac{\partial\left(C_{\eta} H u\right)}{\partial \xi}+\frac{\partial\left(C_{\xi} H v\right)}{\partial \eta}\right]=0 \\
\frac{\partial(H u)}{\partial t}+\frac{1}{C_{\xi} C_{\eta}}\left[\frac{\partial\left(C_{\eta} H u u\right)}{\partial \xi}+\frac{\partial\left(C_{\xi} H u v\right)}{\partial \eta}+H u v \frac{\partial C_{\xi}}{\partial \eta}-H v^{2} \frac{\partial C_{\eta}}{\partial \xi}\right] \\
=-g u \frac{\sqrt{u^{2}+v^{2}}}{C^{2}}-\frac{g H}{C_{\xi}} \frac{\partial Z}{\partial \xi} \\
+\frac{1}{C_{\xi} C_{\eta}}\left[\frac{\partial\left(C_{\eta} H \sigma_{\xi \xi}\right)}{\partial \xi}+\frac{\partial\left(C_{\xi} H \sigma_{\eta \xi}\right)}{\partial \eta}+H \sigma_{\xi \eta} \frac{\partial C_{\xi}}{\partial \eta}-H \sigma_{\eta \eta} \frac{\partial C_{\eta}}{\partial \xi}\right]_{0} \\
\frac{\partial(H v)}{\partial t}+\frac{1}{C_{\xi} C_{\eta}}\left[\frac{\partial\left(C_{\eta} H u v\right)}{\partial \xi}+\frac{\partial\left(C_{\xi} H v v\right)}{\partial \eta}+H u v \frac{\partial C_{\eta}}{\partial \xi}-H u^{2} \frac{\partial C_{\xi}}{\partial \eta}\right] \\
=-g v \frac{\sqrt{u^{2}+v^{2}}}{C^{2}}-\frac{g H}{C_{\eta}} \frac{\partial Z}{\partial \eta} \\
+\frac{1}{C_{\xi} C_{\eta}}\left[\frac{\partial\left(C_{\eta} H \sigma_{\xi \eta}\right)}{\partial \xi}+\frac{\partial\left(C_{\xi} H \sigma_{\eta \eta}\right)}{\partial \eta}+H \sigma_{\eta \xi} \frac{\partial C_{\eta}}{\partial \xi}-H \sigma_{\xi \xi} \frac{\partial C_{\xi}}{\partial \eta}\right]
\end{gathered}
$$

Where, $\zeta$ and $\eta$ are the two orthogonal curvilinear coordinates in the orthogonal curvilinear coordinate system. The finite volume method [5] was used to discretize the above equation, and the simple algorithm is used to solve the discrete equation. In order to avoid the overflow value in the iterative process, the under relaxation technology, block correction technology and progressive scanning ADI method given by Patanker and Splading were used [6].

\subsection{Data}

The latest measured topographic and hydrological data in August 2020 were used in this study. According to the measured topography, the shape of the studied river is mainly U-shaped, and the fluctuation of the river bottom is small. During the hydrological measurement period, the flow of the reach was about $2044 \mathrm{~m}^{3} / \mathrm{s}$. Three Hydrometric sections, MCS01 MCS03, were arranged, whose position was shown in Figure 2.

\section{Results}

\subsection{Model validation}


Table 1 is the results of water level verification. Figure 3 shows the verification of the cross-section velocity distribution. It can be seen from the above that the error of the water level calculated by the mathematical model is small, the calculation flow field is clearly distinguished between the shoals and troughs, and the cross-section velocity distribution well conforms to the actual situation. Generally speaking, the mathematical model has high accuracy and can well reflect the flow movement characteristics of the studied river reach.

Table 1. Water level verification results.

\begin{tabular}{|c|c|c|c|}
\hline Section & Measured $(\mathrm{m})$ & Calculated $(\mathrm{m})$ & Error $(\mathrm{m})$ \\
\hline MCS01 & 17.816 & 17.815 & -0.001 \\
\hline MCS 02 & 17.817 & 17.817 & 0.000 \\
\hline MCS 03 & 17.818 & 17.819 & +0.001 \\
\hline
\end{tabular}

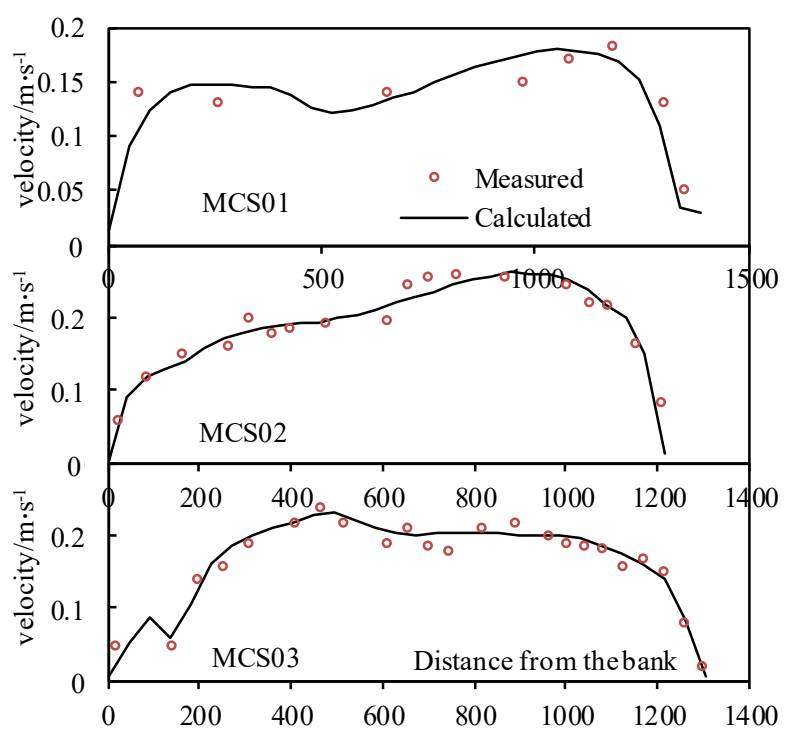

Fig. 3. Map of the cross-section velocity distribution.

\subsection{Flow characteristics}

The characteristics of river flow movement under the flood discharge and bed-forming discharge was calculated and analysed. Among them, the flood discharge is represented by $2 \%$ frequency, and the bedforming discharge is represented by the $5 \%$ frequency, shown as Table 2.

Table 2. Flow conditions of the calculation.

\begin{tabular}{|c|c|c|}
\hline Item & $\mathrm{P}=2 \%$ & $\mathrm{P}=5 \%$ \\
\hline Discharge $\left(\mathrm{m}^{3} / \mathrm{s}\right)$ & 23400 & 20500 \\
\hline Water Level at Bayi Bridge $(\mathrm{m})$ & 22.96 & 22.48 \\
\hline
\end{tabular}

\subsubsection{Water surface}

Figure 4 shows the water surface profile along the reach under different flow rates. It can be seen from the figure that the water surface gradient of the study river reach is small, the average gradient under the frequency of $\mathrm{P}=$ $2 \%$ is about $0.24 / 10000$, and the average gradient under the frequency of $\mathrm{P}=5 \%$ is about $0.20 / 10000$. This is mainly due to the fact that the study section is located at the tail end of Ganjiang River, and the water surface gradient is slower than that in the upper reaches of Ganjiang River under the influence of Poyang Lake. This kind of flow characteristics is easy to cause a smooth water surface, which is easy to lead a flood disaster in this area.

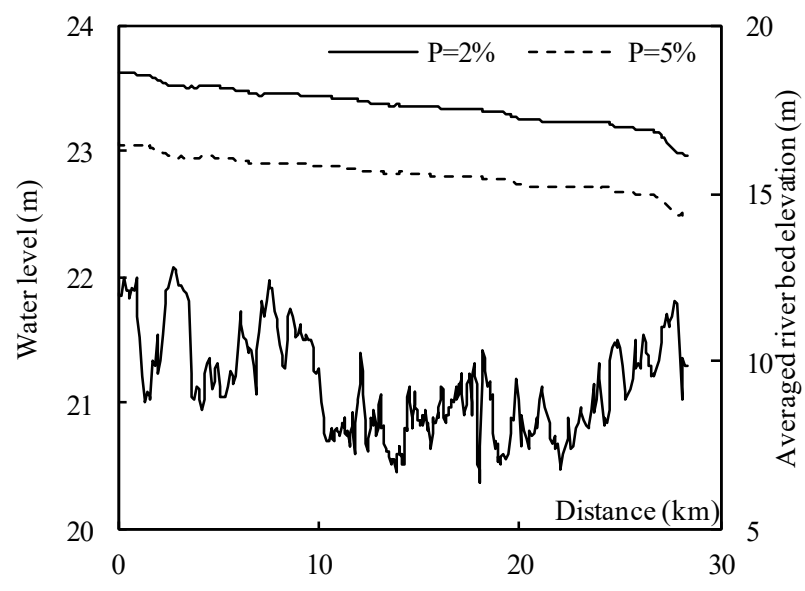

Fig. 4. Map of water surface along the river reach.

\subsubsection{Velocity and flow field}

Figure 5 shows the flow field under different discharge conditions. It can be seen from the figure that, due to the small fluctuation of river bottom elevation, the crosssection is mostly U-shaped, so the cross-section velocity distribution is relatively uniform, and the change of the vertical flow direction is small. At the same time, because the water surface gradient is small and the flow velocity is not large, the sediment is easy to deposit in this area after the flow carries the sediment into the reach. Figure 6 shows the variation of riverbed elevation from 2013 to 2020 . It can be seen from the figure that in recent years, the river reach is mainly at deposition status, which is consistent with the characteristics of slower flow velocity and more stable flow pattern in the engineering reach.

\section{Conclusions}

Based on the mathematical model verified by the latest measured topographic and hydrological data, the flow characteristics in the tail reach of Ganjiang River are calculated and analysed in this paper. The results show that the riverbed of Ganjiang River at the tail end of Nanchang city is relatively flat, the water surface gradient is small, the flow field is relatively smooth, and the flow velocity is small, which is not only easy to cause flood disaster, but also easy to cause riverbed siltation. Therefore, in order to reduce the flood impact of the bridge, more attention should be paid to the control of the water blocking rate of the project in this river section. At the same time, the designed elevation of the bridge should consider the influence of water level elevation caused by riverbed sedimentation in the future, so as to avoid the loss of bridge beam flooding. 


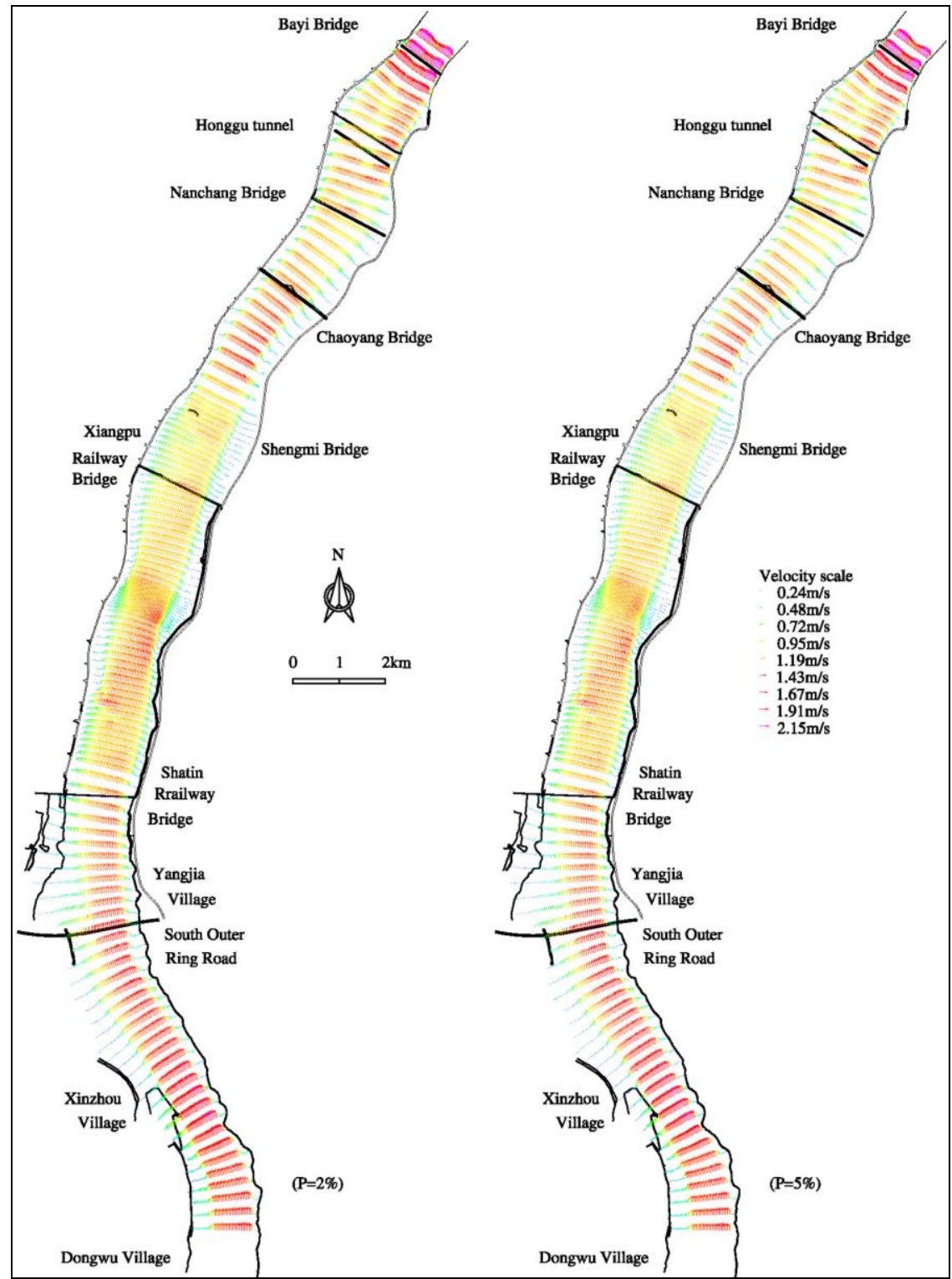

Fig. 5. Map of flow field distribution under different flow rates.

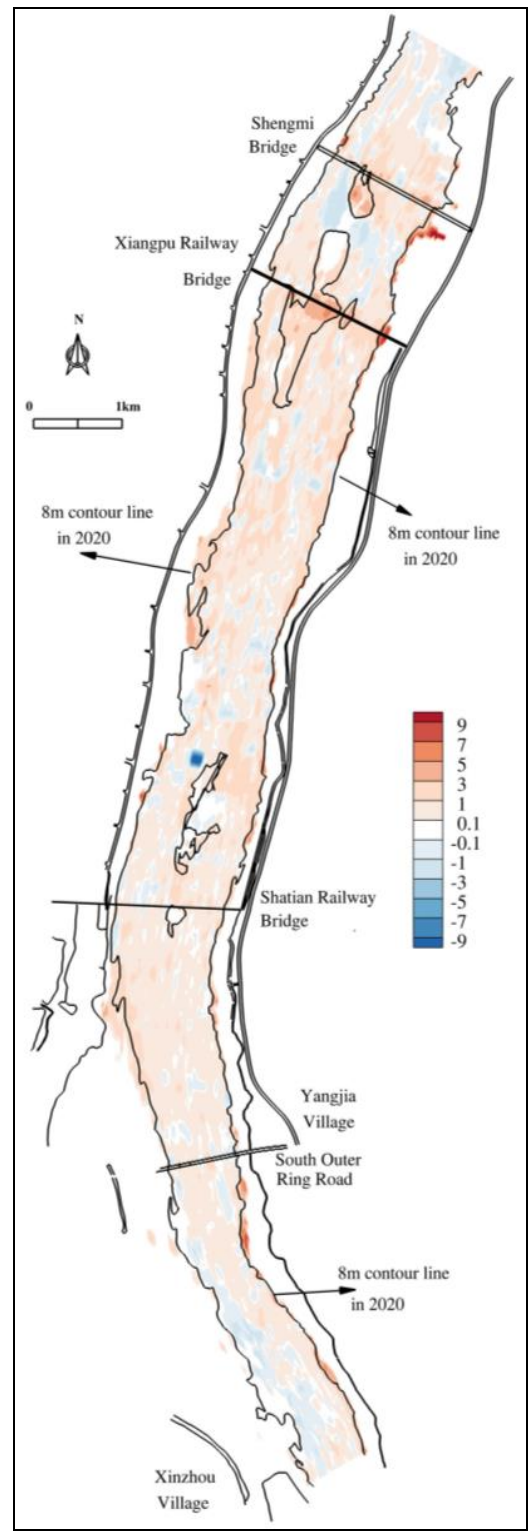

Fig. 6. River bed elevation changes from 2013 to $2020, \mathrm{~m}$

\section{Acknowledgments}

This work was supported by National Key R\&D Program of China 2017YFC0405306, and the Second Stage Research Project of Key Technologies for Scientific Operation of the Three Gorges Reservoir (0714167).

\section{References}

1. Reply of the State Council on the overall urban planning of Nanchang City. Bulletin of the State Council of the people's Republic of China, 35(2012)

2. SHEN Sunping. Analysis of Impact of Bridges engineering on River Based on 2-D Numerical Modal. GUANGDONG WATER RESOUR CES AND HYDROPOWER, 10, (2019)
3. QIAO Lian-peng, ZHENG Xiang-long, ZHANG De-xi. Comprehensive Analysis and Evaluation of the Influence of Cross-River Bridge on River Flood Control. Water Conservancy Science and Technology and Economy, 6, 25 (2019)

4. LIU Xuecong, GENG pengcheng, CAO li, S Xiuling. Simulation of the influence of river crossing bridge on river regime by MIKE 21 . YELLOW RIVER, 42,1 (2020)

5. JIANG Youxiang, LIU Yang, WEN Cen. Application of two dimensional flow mathematical model based on finite volume method in bridge flood control evaluation. China Water Transport, 19, 10(2019)

6. Patankar, S.V, Spalding, D.B. A Calculation Procedure for Heat, Mass and Momentum Transfer in Three-Dimensional Parabolic Flows. International Journal of Heat and Mass Transfer, 15 (1972) 